\title{
COMPARISON OF TDMA VERSUS CDMA FOR PACKET TRANSMISSION SCHEMES IN CIRCUIT-MODE
}

\author{
Jeetendra Sant Vinod Sharma * \\ Dept of Electrical Engg \\ Indian Institute of Science \\ Bangalore-560012, INDIA
}

July 11,1998

\begin{abstract}
We compare the performance of a TDMA multiple access scheme with a CDMA multiple access scheme when both are used for packet transmission in circuit-mode. First we consider a more general model of slotted ALOHA protocol on a channel with capture effect. Both TDMA and CDMA are shown to be special cases of this model. There are $M<\infty$ users each with an infinite buffer. If in a slot, $i$ packets are transmitted, then probability of a successful reception of a packet is $q_{i}$. We state our results for this model and apply them to the special cases of TDMA and CDMA. We compare their stability regions, the rates of convergence to stationary distribution and the delay moments. We find the range of the number of users and their arrival rates for which CDMA has higher throughput and lower delay moments as compared to the TDMA system.
\end{abstract}

\section{Introduction}

In this paper, we present some results on performance analysis of a CDMA (code division multiple access) system and its comparison with a TDMA (time division multiple access) system when both are used for packet transmission in circuit-mode. Each of the transmitting nodes is allotted a channel permanently in both the systems; hence the name 'circuitmode' [2]. In both the systems there are a fixed, finite number of users each with an infinite buffer. For the CDMA system, the channel is assumed to be slotted with mutually aligned slots between different users. If $i$ nodes transmit packets in a slot, a packet is successfully received with probability $q_{i}$. Unsuccessful packets are retransmitted immediately in the next slot. For the TDMA system, the channel is divided in frames, with each transmitting node getting a slot in successive frame. Any transmitted packet is successful with probability one.

*email:- vinod@ece.iisc.ernet.in

0-7803-4984-9/98/\$10.00@1998 IEEE.
We compare the stability regions, rates of convergence and conditions for finiteness of moments for the two systems when the arrival traffic is general stationary ergodic or regenerative. This generality is important for the traffic generated by packetized voice and video. For i.i.d. arrivals, we also provide comparison of the delay moments via explicit expressions. Similar comparison can be made for Markov modulated arrivals via computational algorithms. This will be provided in a future work.

The capacity comparison of the two schemes for circuit-switched systems has been a matter of debate for quite some time [4]. For packetized systems, the comparison should include packet throughput per node and the moments of sojourn time in the buffer, as considered in this paper. The past work in this field either assumes very simple, unrealistic models for the arrival process and the channel ([8], $[10])$, or relies exclusively on simulations ([1], [5], [9]). In contrast to these, the emphasis in this paper is on analytical comparison.

Recently, we have formulated another model [7] where the mobility of users and the fading process of a channel have also been taken into account. However, explicit comparisons become more difficult.

The rest of the paper is planned as follows. Section 2 states the comparison results on stability, rates of convergence and delay moments. Section 3 gives simulation results. Section 4 states the conclusions.

\section{The Models and their Com- parison}

In both the CDMA and the TDMA systems, there are $M<\infty$ nodes each having an infinite buffer. The packets generated at each of the nodes are stored in the buffer and transmitted on the channel till each of the packets is successfully received. Now we mention the slot structure and the channel model in the two cases. For CDMA, the channel is assumed slotted with the slot duration equal to the packet transmis- 
sion time. If a node has a packet in its buffer at the beginning of a slot, it transmits a packet in that slot. If $i$ packets are transmitted in a slot, then the probability of successful reception of a packet is $q_{i}$, with the successes for different packets being mutually independent of each other. We assume that

$$
q_{1} \geq q_{2} \geq \cdots \geq q_{M}>0 .
$$

The assumption corresponds to the fact, that as more number of packets are transmitted in a slot, there is greater interference to each of the packets and hence smaller probability of it being received successfully. For some typical system parameters, the $q_{i}$ 's are computed in the next section. For TDMA, the channel is assumed to be divided in frames, with each frame consisting of $M$ slots and each slot duration is equal to the packet transmission time. The $i^{t h}$ slot in every frame is allotted to the $i^{\text {th }}$ node (generalization to the case where different users are allocated different number of slots in a frame will require simple modifications in our results). If a node has a packet available in its buffer at the beginning of its slot in any frame, it transmits the packet in that slot. The packet is assumed to be successfully received with probability one.

Now we specify the exact connection between the two systems. The bandwidth offered to both the systems is the same and both have the same spectral efficiency per cell. In CDMA, each cell uses the entire bandwidth, whereas in TDMA, each cell uses $1 / R$ times the total bandwidth, where $R$ is the cell reuse factor. The spread factor for CDMA is assumed to be $S$. This implies that for the same packet size, the packet transmission time for CDMA is $S / R$ times that for TDMA and hence the CDMA slot length is $S / R$ times the TDMA slot length. We assume $S / R$ is an integer (this assumption can be relaxed), which allows us to assume a common arrival process to both the systems as described in the notations below -

$$
\begin{aligned}
x_{k}^{B}(i)= & \text { number of arrivals at the } i^{\text {th }} \text { queue in } \\
& \text { the } k^{t h} \text { basic TDMA slot. } \\
x_{k}^{T}{ }_{k}(i)= & \text { number of arrivals at the } i^{t h} \text { queue in } \\
& \text { the } k^{t h} \text { TDMA frame (with the frame } \\
& \text { boundary considered as the beginning } \\
& \text { of the } i^{t h} \text { slot of the frame). } \\
x^{C}{ }_{k}(i)= & \text { number of arrivals at the } i^{\text {th }} \text { queue in } \\
& \text { the } k^{t h} \text { CDMA slot. }
\end{aligned}
$$

Then

$$
\begin{aligned}
& x^{C}{ }_{k}(i)=\sum_{l=k S / R}^{((k+1) S / R)-1} x^{B}{ }_{l}(i) \quad \text { and } \\
& x^{T}{ }_{k}(i)=\sum_{l=k M}^{(k+1) M-1} x^{B}{ }_{l}(i) .
\end{aligned}
$$

Note that if $\left\{x^{B}{ }_{k}(i)\right\}$ is i.i.d. , Markov modulated or stationary ergodic, then respectively the same holds for both $\left\{x^{C}{ }_{k}(i)\right\}$ and $\left\{x^{T}{ }_{k}(i)\right\}$. Also $\lambda^{C}(i)=$ $(S / R) \lambda^{B}(i)$ and $\lambda^{T}(i)=M \lambda^{B}(i)$.

Let $z^{T}{ }_{k}(i)$ and $z^{C}{ }_{k}(i)$ denote the total number of packets at the beginning of the $k^{\text {th }}$ TDMA frame and the $k^{\text {th }}$ CDMA slot respectively at the $i^{\text {th }}$ node. Then

$$
\begin{aligned}
& z_{k+1}^{T}(i)=\left(z_{k}^{T}(i)-T_{k}^{T}(i)\right)^{+}+x_{k}^{T}(i) \quad \text { and } \\
& z^{C+1}(i)=\left(z_{k}{ }_{k}(i)-T_{k}^{C}(i)\right)^{+}+x_{k}{ }_{k}(i)
\end{aligned}
$$

where $\left\{T_{k}^{C}(i), k \geq 1\right\}$ are $\{0,1\}$ valued and given $\ell\left(z^{C}{ }_{k}(i)\right), T^{C}{ }_{k}(i)$ is independent of anything else and has distribution $-\mathbb{P}\left(T^{C}{ }_{k}(i)=1 \mid \ell\left(z^{C}{ }_{k}(i)\right)=j\right)=$ $q_{j}=1-\mathbb{P}\left(T^{C}{ }_{k}(i)=0 \mid \ell\left(z^{C}{ }_{k}(i)\right)=j\right)$, where $\ell\left(z^{C}{ }_{k}\right)$ is the cardinality of $\left\{z^{C}{ }_{k}(j)>0: 1 \leq j \leq M\right\}$. For TDMA, $\mathbb{P}\left(T_{k}^{T}(i)=1\right)=1$.

Note that the queuelength equation for TDMA is a special case of that for CDMA; for TDMA all $q_{i}$ 's are one. Hence the delay bounds obtained below for CDMA are also applicable to TDMA with the appropriate substitutions. It is known that if $\left\{x^{T}{ }_{k}(i)\right\}$ is stationary ergodic then TDMA is stable (i.e. $\left\{z^{T}{ }_{k}(i), i=1, \ldots, M\right\}$ has a proper unique stationary distribution) if

$$
\lambda^{T}(i)<1 \text { for all } i \text {. }
$$

We now discuss the stability of the CDMA system. The following results are new and are obtained in [6]. We state the results without proof.

Assume that for $i=1, \ldots, M,\left\{x^{C}{ }_{k}(i), k \geq 0\right\}$ is stationary, ergodic and is independent of $\left\{x_{k}{ }_{k}(j)\right\}$, $j \neq i$. Without loss of generality, for the rest of the paper assume that

$$
0<\lambda_{1} \leq \lambda_{2} \cdots \leq \lambda_{M} .
$$

We define system $\mathcal{L}^{(i)}$ in which queue $i+1$ to queue $M$ are set to infinity (so these nodes are always transmitting packets on the channel) and the first $i$ queues are allowed to evolve with the channel operating as before. We denote the original system by $\mathcal{L}$. In the following, stability of a system means the existence of a proper stationary distribution to which the queue length process converges in total variation starting from empty state. Also, stability of $\mathcal{L}^{(\hat{i})}$ refers to the stability of only the first $i$ queues.

Define recursively $\tilde{\rho}_{l}^{(i)}$ and $\tilde{r}_{j}$ as follows:

$$
\begin{gathered}
\tilde{\rho}_{0}^{(1)}=\lambda_{1} / q_{M}, \quad \tilde{\rho}_{1}^{(1)}=1-\tilde{\rho}_{0}^{(1)}, \\
\tilde{\rho}_{l}^{(i+1)}=\tilde{\rho}_{l}^{(i)} \mu_{i+1}+\tilde{\rho}_{l-1}^{(i)}\left(1-\mu_{i+1}\right), \\
\tilde{r}_{1}=q_{M}, \quad \tilde{r}_{i+1}=\sum_{l=0}^{i} \tilde{\rho}_{l}^{(i)} q_{M-l}, i=1,2, \ldots, M-1,
\end{gathered}
$$

where $\mu_{i}=\lambda_{i} / \tilde{r}_{i}$. The following theorem is proved in [6]. 
Theorem $1 \mathcal{L}^{(i)}$ is stable if $\lambda_{j}<\tilde{r}_{j}, 1 \leq j \leq i$. In particular, $\mathcal{L}$ is stable if $\lambda_{j}<\tilde{r}_{j}$, for all $j$.

If the system is stable for the case of regenerative arrival process, then there exists a unique stationary distribution for the queue length process, and the convergence to it holds starting from any initial state.

Stability of $\mathcal{L}^{(i)}$ in the above theorem implies that, irrespective of the traffic to queues $i+1, \ldots, M$, the queue lengths at the first $i$ queues will remain (stochastically) bounded if they start empty at time $t=0$.

In [6], we also have sufficient conditions of the form $\lambda_{j}<r_{j}, 1 \leq j \leq i$, which imply the stability of system $\mathcal{L}^{(i)}$. These have been shown to be close to the necessary conditions for Markov modulated arrival process, in the sense that if $\lambda_{j}>r_{j}$, for some $j, 1 \leq j \leq i$, then $\mathcal{L}^{(i)}$ is unstable. $r_{j}, j \geq 3$, have to be computed by simulating $\mathcal{L}^{(j-1)}$. The conditions in theorem 1 above are not close to necessary i.e., $\tilde{r}_{j} \leq r_{j}$, where the inequality could be strict in some cases. The advantage is that there is no need for any simulations to compute $\tilde{r}_{j}$. We have found via simulations that $\tilde{r}_{j}$ 's are close to $r_{j}$ 's in a vast number of cases. We have also proved this closeness analytically under both heavy and light traffic conditions.

Now we consider the case when all the nodes have the same arrival rate. In this case $\lambda^{C}<q_{M}$ is necessary for the stability of the CDMA system, whereas $\lambda^{T}<1$ is necessary for the stability of the TDMA system. Figure 1 shows the maximum achievable throughput (in terms of the number of packets per basic TDMA slot per node) versus the number of transmitting nodes for the two systems. The procedure used to obtain the capture probabilities for the above figures is explained in section 3 , where $\gamma$ refers to the path loss coefficient. A higher $\gamma$ leads to lower interference from outside the cell and hence improves the CDMA system performance [1]. For a fixed spread factor $S, q_{i}$ is equal to 1 upto a certain point (say $i_{0}$ ) and then sharply falls to zero. $i_{0}$ dependes on the spread factor. For example, for $S=63$ and $\gamma=4, i_{0}=39$. Hence if $M \leq i_{0}$, CDMA is stable for $\lambda^{B}<R / S$. This explains a flat curve upto $i_{0}$ which then goes to zero. TDMA is stable if $\lambda^{B}<1 / M$. So the two curves cross for the first time at $M=S / R$. For $M<S / R$, TDMA supports higher arrival rates. For $M>S / R$ such that $q_{M} \geq S /(R M)$, CDMA supports higher arrival rates. Again for $M>S / R$ such that $q_{M}<S /(R M)$, TDMA gives superior throughput. Also note that, although the plots show maximum achievable throughput, any system operating close to that curve though stable faces a very high delay. So the actual allowed throughput in a realistic system would be less depending upon the delay constraint imposed.

From [6], we also obtain the rates of convergence to stationary distribution for a regenerative arrival process. Let $\left\{x_{k}(i)\right\}$ be regenerative with regeneration length distributed as $\{\tau(i)\}$. If $\mathbb{E}\left[(\tau(i))^{s}\right]<\infty$ and $\operatorname{Sup}_{i, k} \mathbb{E}\left[\left(x_{k}(i)\right)^{s} \mid \mathcal{F}_{k-1}\right]<\infty, s \geq 1$, for all $k$, where $\left\{\mathcal{F}_{k}\right\}$ is a filteration and $x_{k}(i)$ is measurable $\mathcal{F}_{k}$, then for both CDMA and TDMA systems,

$$
\operatorname{Sup}_{A}\left|\mathbb{P}\left(\left(z_{k}, x_{k}\right) \in A\right)-\pi(A)\right|<C k^{1-s}
$$

for some constant $C$ ( $C$ may be different for the two systems). Thus both the systems have the same set of sufficient conditions for polynomial rates of convergence.

Now we state the results on mean sojourn time for the two systems. Consider a slotted single server queue in which both the arrival and the service time proesses are i.i.d. and are mutually independent. Let the number of arrivals in a slot be distributed as $x$ and the service time of each arrival be Geometric with mean $1 / q$ slots. Then the mean sojourn time of an arrival is given (from [3]) by $\frac{(1-q) \mathbb{E}[x]+\operatorname{var}(x)}{2 \mathbb{E}[x](q-\mathbb{E}[x])}+\frac{1}{2}$. Now define

$d(q, K, i)=K\left[\frac{(1-q) \mathbb{E}\left[x^{B}(i)\right]+\operatorname{var}\left(x^{B}(i)\right)}{2 \mathbb{E}\left[x^{B}(i)\right]\left(q-K \mathbb{E}\left[x^{B}(i)\right]\right)}+\frac{1}{2}\right]$.

Then it can be proved that for TDMA, the mean delay is

$$
\mathbb{E}\left[D^{T}(i)\right]=d(1, M, i),
$$

whereas the bounds for CDMA are

$$
d\left(q_{1},(S / R), i\right) \leq \mathbb{E}\left[D^{C}(i)\right] \leq d\left(q_{M},(S / R), i\right) .
$$

We have noted before that $q_{i}=1$ for $i \leq i_{0}$ and then falls to zero, where $i_{0}$ depends on the spread factor $S$ and the path loss coefficient $\gamma$. Also we always have $q_{1}=1$. Hence if $M \leq i_{0}$, the upper and lower bounds match for CDMA. In this case, if $M<S / R$, then TDMA gives a better delay performance, which can be found by straight comparison of the above two expressions. From simulations, we also find that the actual mean delay for CDMA is close to its lower bound. In such a case, for $M>S / R$, CDMA has lower mean delay. This is because lower bound for CDMA is fixed irrespective of $M$ (of course, $M$ has to be within the stability region), whereas $\mathbb{E}\left[D^{T}\right] \rightarrow$ $\infty$ as $M \rightarrow 1 / \mathbb{E}\left[x^{B}\right]$.

It is also possible to compare the moments of sojourn times in the two systems if the arrival traffic to each queue is Markov modulated. For this, instead of explicit formula, we have computational algorithms. This comparison will be provided elsewhere. 


\section{Simulations}

For simulations, we first specify the parameters $R, S$, $\gamma$ and $q_{i}$. We take $R=7$, which is commonly taken for hexagonal cells. We make comparison among CDMA systems with $S=63$ or 126 and $\gamma=3$ or 4. We then take $S=63$ and $\gamma=4$ for a CDMA system and compare it with TDMA. To find $q_{i}$ 's for the purpose of simulations, we use the same model as in [1], which uses the standard Gaussian Approximation(SGA). Assuming that the multiple access interference (MAI) is Gaussian, and using simple correlation receivers, probability of bit error $P_{e}$ is approximately

$P_{e} \approx Q(\sqrt{\mathrm{SNR}})$ where $Q(x)=\frac{1}{\sqrt{2 \pi}} \int_{x}^{\infty} e^{-u^{2} / 2} d u$.

Assume random direct sequences, with 0.5 prob of chip value \pm 1 . Also assume perfect power control, so all packets are received by their respective Base stations at equal power level $P_{0}$. In this case, SNR is obtained as SNR $=3 P_{0} S /\left(I_{1}+I_{2}\right)$, where $I_{1}$ and $I_{2}$ are interferences faced by a tagged transmission from within the same cell and from the neighbouring cells respectively. There are many ways to estimate $I_{2}$ accurately ([1], Appendix D in [5], Appendix in [9]). For our comparison purposes, we use the method in [1] for convenience. It assumes $K$ transmitters transmitting in the reference as well as the neighbouring cells. Then we obtain $I_{1}$ and $I_{2}$ as

$$
I_{1}=(K-1) P_{0}, \quad I_{2}=I_{\text {ntercell }} K P_{0},
$$

where $I_{\text {ntercell }}=0.749$ and 0.37 respectively for a path loss coefficient $\gamma=3$ and 4 . In a packet of $L$ bits, we assume upto $t$ correctable errors. Then the packet success probability is

$$
q_{K}=\sum_{i=0}^{i=t}\left(\begin{array}{c}
L \\
i
\end{array}\right)\left(P_{e}\right)^{i}\left(1-P_{e}\right)^{L-i}
$$

Thus the capture probabilities $q_{i}$ 's can be computed. We assume $L=1024$ bits with $t=60$ correctable errors. These are close to the ones in [5], in which a packet length of 1023 bits is chosen, which can support an ATM cell after BCH block coding.

We use mutually independent i.i.d. arrival processes to each node with the following two distributions:

Distribution $1 . \quad \mathbb{P}(x=1)=0.008, \mathbb{P}(x=10)=$ 0.0008 and $\mathbb{P}(x=0)=1-\mathbb{P}(x=1)-\mathbb{P}(x=10)$.

Distribution 2. $\quad \mathbb{P}(x=1)=0.01, \mathbb{P}(x=2)=$ $0.01, \mathbb{P}(x=5)=0.004, \mathbb{P}(x=20)=0.00139$ and $\mathbb{P}(x=0)=1-\mathbb{P}(x=1)-\mathbb{P}(x=2)-\mathbb{P}(x=5)-$ $\mathbb{P}(x=20)$.
For distribution $1, \mathbb{E}[x]=0.016, \operatorname{var}(x)=0.0877$, $\mathbb{E}\left[x^{3}\right]=0.808$, whereas for distribution $2, \mathbb{E}[x]=$ $0.0778, \operatorname{var}(x)=0.7000, \mathbb{E}\left[x^{3}\right]=11.71$. Despite this vast difference in variability, the comparison follows a similar trend for both of them. Figure 2 confirms the fact that a higher $\gamma$ results in a better performance of a CDMA system. A similar conclusion is drawn in [1] and [9]. It also shows that for a fixed bandwidth allocation, a higher spread factor leads to a higher mean delay, although it can also support a larger number of nodes. This also follows from our lower and upper bounds in (6) on mean delays. Figure 3 and 4 show that the upper and lower bounds on mean delay for CDMA match over a wide range. Also the lower bound is quite close to the simulated delay. The two figures also show mean delay comparison between the two systems. For $M>9$ (which is equal to $S / R$ ) CDMA has a lower mean delay. This was theoretically justified in the previous section.

In all the figures, the mean delay is expressed in terms of the TDMA slots. To get an idea about the corresponding delay in seconds, consider a total bandwidth of $14 \mathrm{MHz}$ and a spectral efficiency of $1 \mathrm{bit} / \mathrm{s} / \mathrm{Hz}$ per cell for both the systems. Hence $R=7$ means that each cell in the TDMA sys tem has a channel rate of $2 \mathrm{Mbits}$ per second. So a packet of 1024 bits can be transmitted (neglecting the guard bands) in a TDMA slot of $0.512 \mathrm{~ms}$. Suppose a voice packet tolerates a delay of $40 \mathrm{~ms}$. Then in terms of TDMA slots it comes to around 78 slots.

For the above cases of arrival processes, we also have comparison of the second moments of delays, which are not given here because of space constraint. The plots are qualitatively similar to the plots for the first moment.

\section{Conclusions}

A comparison of the performance of a TDMA system with that of a CDMA system was presented. In general, the performance of such multiple access schemes is a complicated function of the system parameters such as spread factor $S$, environmental variables e.g., the path loss coefficient $\gamma$, the number of users $M$ loading the system and their traffic characteristics. Our study gives some idea about their interdependence.

The following results were obtained. We proved, under the assumption of mutually independent stationary ergodic arrival processes to each node, that the TDMA system is stable if $\lambda^{B}{ }_{i}<1 / M$, whereas the CDMA system is stable if $\lambda^{B}{ }_{i}<\tilde{r}_{i} /(S / R)$, For the particular case of equal arrival rate to each node, CDMA is stable if $\lambda^{B}<q_{M} R / S$. This leads to the 
further conclusion that for very small $M(M<S / R)$ and for very large number of transmitting nodes $M$ ( $M$ such that $M>S / R$ and $q_{M}<S /(R M)$ ), TDMA gives higher throughput, whereas over a very wide range in the middle, CDMA gives higher throughput. For regenerative arrival sequences, we have proved that the moment conditions for polynomial rate of convergence to stationary distribution are the same for both the systems. For the case of mutually independent i.i.d. arrival processes to each node, we obtained the exact expression for the mean sojourn time in TDMA, which is given in (5). Bounds were obtained for CDMA which are given in (6). From these results we concluded that CDMA has lower delay moments for $M>S / R$ and for $M$ large (within the stability region of CDMA), has a much superior performance compared to TDMA. Therefore, in particular, for $M>S / R$ and $q_{M} \geq S / R M$, CDMA has lower mean delay as well as higher capacity. Also, the CDMA system has a better performance for a higher path loss coefficient. We also observed that a higher spread factor results in a higher mean delay, although it is capable of supporting larger throughput.

\section{References}

[1] A. E. Brand and A. H. Aghvami. Performance of a joint CDMA/PRMA protocol. IEEE Journal on Selected Areas in Communication, 14(9):1698-1706, Dec 1996.

[2] A. DeSimone and S. Nanda. Wireless data : Systems, standards, services. Wireless Networks, 1:241-253, 1995.

[3] N. D. Gangadhar. Analysis of discrete-time queves with applications to ATM based BISDNs. Master's thesis, Dept of EE, Indian Institute of Science, Bangalore, India, 1994.

[4] K. S. Gilhousen, I. M. Jacobs, R. Padovani, A. J. Viterbi, L. A. Weaver, Jr., and C. E. Wheatly. On the capacity of a cellular CDMA system. IEEE Transactions on Vehicular Technology, 40(2):303--312, May 1991.

[5] Z. Liu, M. J. Carol, M. E. Zarki, and K. Y. Eng. Channel access and interference issues in multi-code DS-CDMA wireless packet (ATM) networks. Wireless Networks, 2:173-193, 1996.

[6] J. Sant and V. Sharma. Performance analysis of a slotted ALOHA protocol with capture channel. submitted.
[7] V. Sharma and J. Sant. Performance analysis of a multiple access scheme on mobility-dependent nonstationary fading channels. In 2nd Canadian Conference on Broadband Research, Ottawa, 1998.

[8] L. Tan and Q. T. Zhang. A reservation random-access protocol for voice/data integrated spread-spectrum multiple-access systems. IEEE Journal on Selected Areas in Communication, 14(9):1717-1727, Dec 1996.

[9] N. D. Wilson, R. Ganesh, K. Joseph, and D. Raychaudhuri. Packet CDMA versus dynamic TDMA for multiple access in an integrated voice/data PCN. IEEE Journal on Selected Areas in Communication, 11(6):870-884, Aug 1993.

[10] Z. Zhang and Y. J. Liu. Performance analysis of multiple access protocols for CDMA cellular and personal communication services. In Proc. IEEE INFOCOM, pages 1214-1220, 1993. 


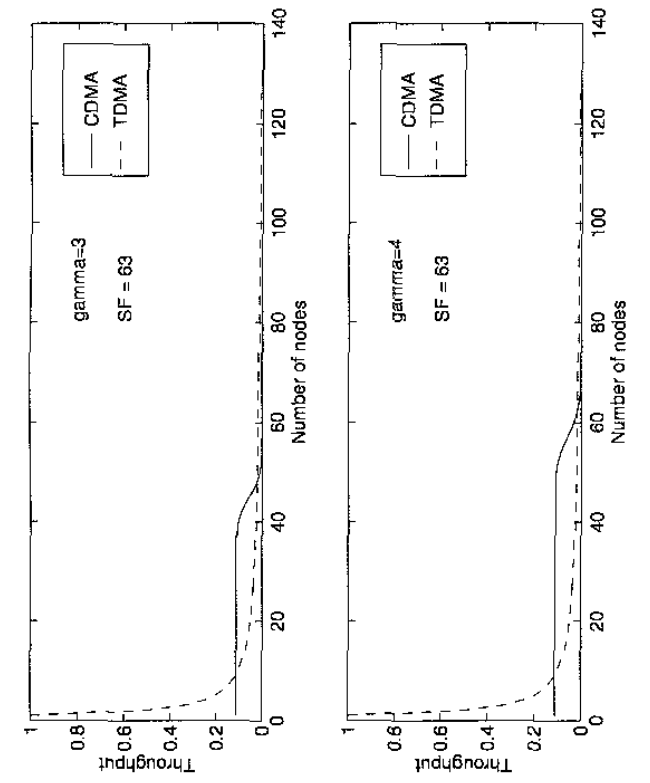

Figure 1: Maximum achievable throughput in terms of number of packets per TDMA slot per node vesus total number of transmitting nodes. $\gamma$ is the path loss coefficient and SF is the spread factor.

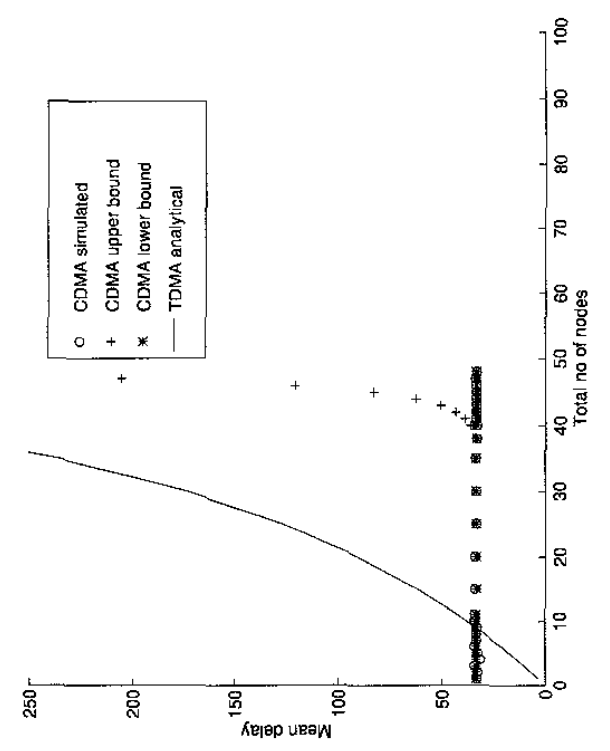

Figure 3: Mean delay comparison of a TDMA system with a CDMA system using probability distribution 1 with $S=63$ and $\gamma=4$

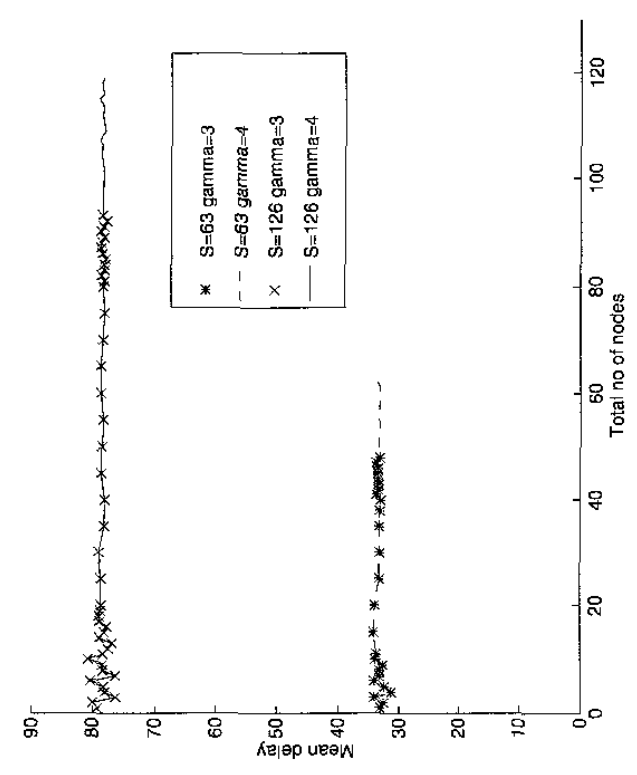

Figure 2: Delay comparison between CDMA systems using distribution 1 with different path loss coefficients and spread factors.

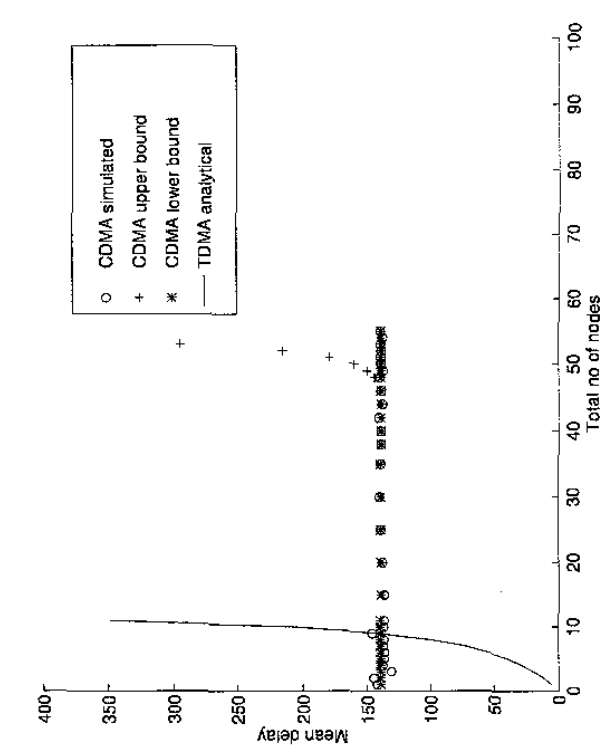

Figure 4: Mean delay comparison of a TDMA system with a CDMA system using probability distribution 2 with $S=63$ and $\gamma=4$ 